\title{
Lanostane Triterpenes Isolated from Antrodia heteromorpha and Their Inhibitory Effects on RANKL-induced Osteoclastogenesis
}

Jaeyoung Kwon, ${ }^{\dagger}$ Hyaemin Lee, ${ }^{\dagger}$ Yeo Dae Yoon, ${ }^{\ddagger}$ Bang Yeon Hwang, ${ }^{\S}$ Yuanqiang Guo, ${ }^{\perp}$ Jong Soon Kang, ${ }^{\star}$ Jae-Jin Kim,, and Dongho Lee ${ }^{*} \dagger$

${ }^{\dagger}$ Department of Biosystems and Biotechnology, College of Life Sciences and Biotechnology, Korea University, Seoul 02841, Republic of Korea

*Bio-Evaluation Center, Korea Research Institute of Bioscience and Biotechnology, Cheongju 28116, Republic of Korea

${ }^{\S}$ College of Pharmacy, Chungbuk National University, Cheongju 28644, Republic of Korea

${ }^{\perp}$ State Key Laboratory of Medicinal Chemical Biology, College of Pharmacy, and Tianjin Key Laboratory of Molecular Drug Research, Nankai University, Tianjin 300353, People's Republic of China

${ }^{\|}$Division of Environmental Science and Ecological Engineering, College of Life Sciences and Biotechnology, Korea University, Seoul 02841, Republic of Korea 


\section{Supplementary data}

\section{List of supplementary data}

S1. ${ }^{1} \mathrm{H}$ NMR spectrum $\left(500 \mathrm{MHz}, \mathrm{CDCl}_{3}\right)$ of antrolactone A (1).

S2. ${ }^{13} \mathrm{C}$ NMR spectrum $\left(125 \mathrm{MHz}, \mathrm{CDCl}_{3}\right)$ of antrolactone $\mathrm{A}(\mathbf{1})$.

S3. HSQC NMR spectrum $\left(\mathrm{CDCl}_{3}\right)$ of antrolactone A (1).

S4. HMBC NMR spectrum $\left(\mathrm{CDCl}_{3}\right)$ of antrolactone A (1).

S5. COSY NMR spectrum $\left(\mathrm{CDCl}_{3}\right)$ of antrolactone $\mathrm{A}(\mathbf{1})$.

S6. NOESY NMR spectrum $\left(\mathrm{CDCl}_{3}\right)$ of antrolactone A (1).

S7. HRESIMS spectrum of antrolactone A (1).

S8. ${ }^{1} \mathrm{H}$ NMR spectrum $\left(500 \mathrm{MHz}, \mathrm{CDCl}_{3}\right)$ of antrolactone B (2).

S9. ${ }^{13} \mathrm{C}$ NMR spectrum $\left(125 \mathrm{MHz}, \mathrm{CDCl}_{3}\right)$ of antrolactone B (2).

S10. HSQC NMR spectrum $\left(\mathrm{CDCl}_{3}\right)$ of antrolactone B (2).

S11. HMBC NMR spectrum $\left(\mathrm{CDCl}_{3}\right)$ of antrolactone B (2).

S12. COSY NMR spectrum $\left(\mathrm{CDCl}_{3}\right)$ of antrolactone B (2).

S13. NOESY NMR spectrum $\left(\mathrm{CDCl}_{3}\right)$ of antrolactone B (2).

S14. HRESIMS spectrum of antrolactone B (2).

S15. ${ }^{1}$ H NMR data of compound 3 .

S16. BMMs were treated with vehicle or indicated concentrations of $\mathbf{1}-\mathbf{3}$ in the presence of RANKL and M-CSF for 4 days. Cell viability was measured by XTT assay. 
S1. ${ }^{1} \mathrm{H}$ NMR spectrum $\left(500 \mathrm{MHz}, \mathrm{CDCl}_{3}\right)$ antrolactone A (1).

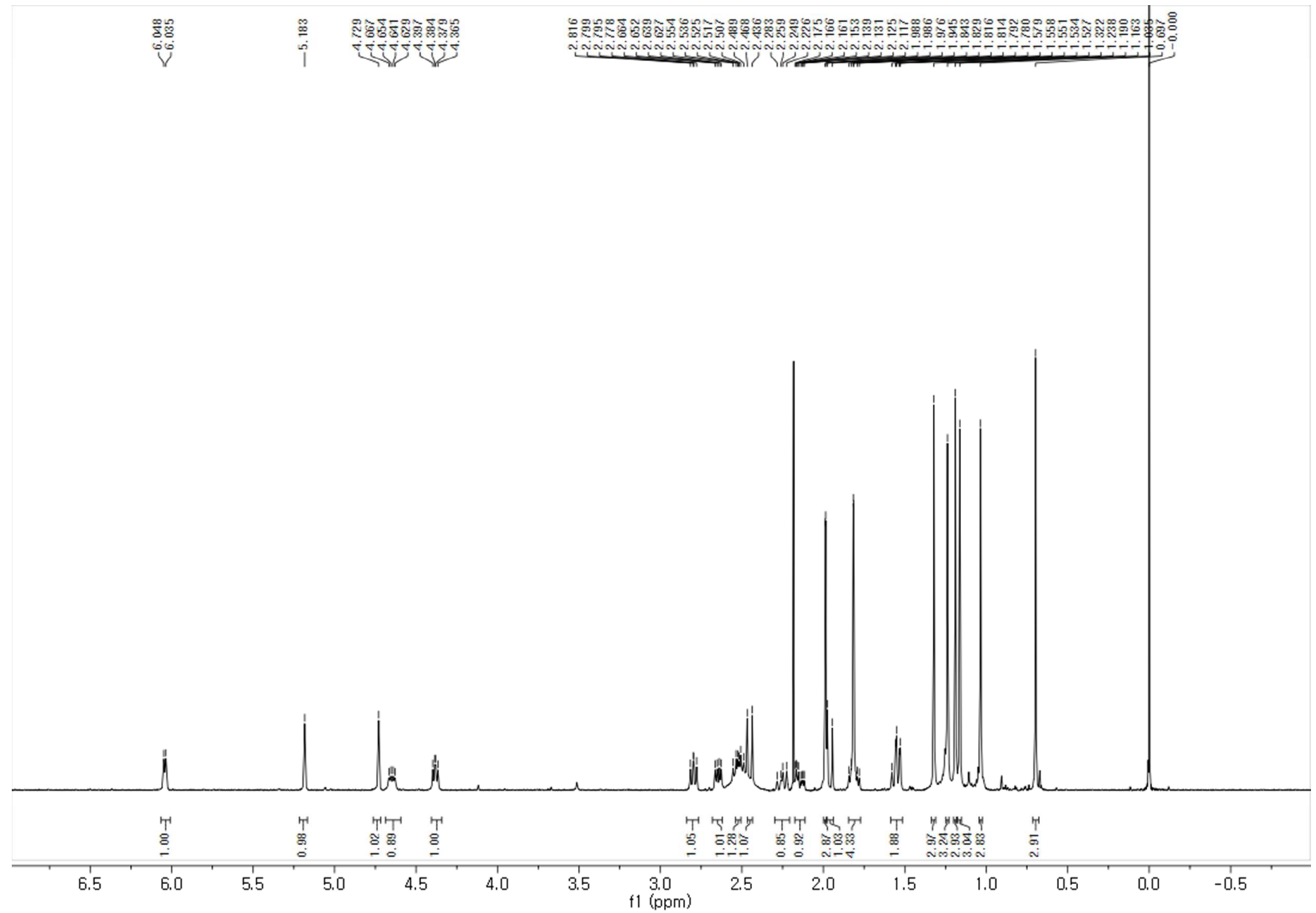


S2. ${ }^{13} \mathrm{C}$ NMR spectrum $\left(125 \mathrm{MHz}, \mathrm{CDCl}_{3}\right)$ antrolactone $\mathrm{A}(\mathbf{1})$.

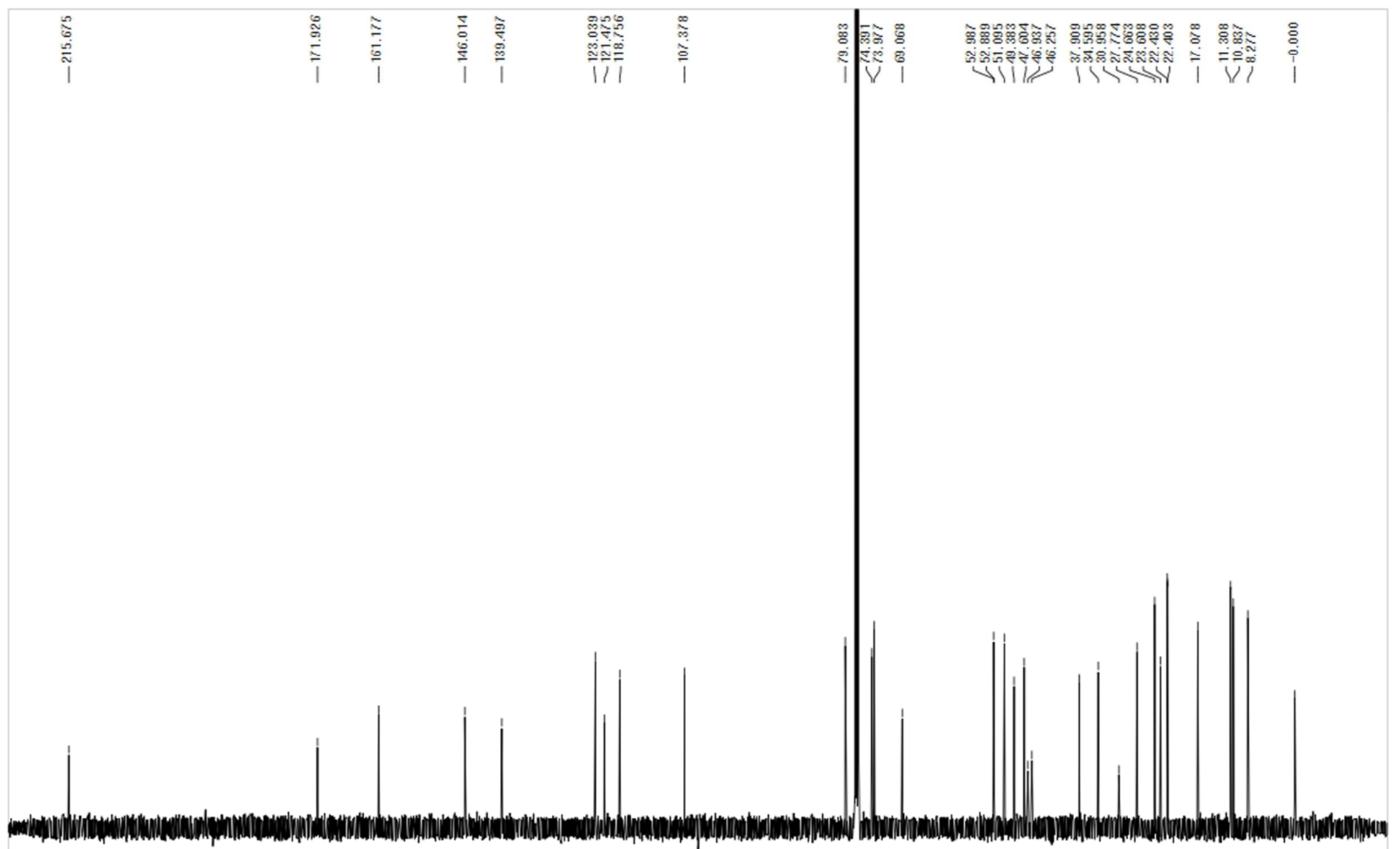

$\begin{array}{lllllllllllll}220 & 210 & 200 & 190 & 180 & 170 & 160 & 150 & 140 & 130 & 120 & 110 & 100\end{array}$
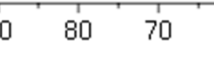

60 
S3. HSQC NMR spectrum $\left(\mathrm{CDCl}_{3}\right)$ antrolactone $\mathrm{A}(\mathbf{1})$.

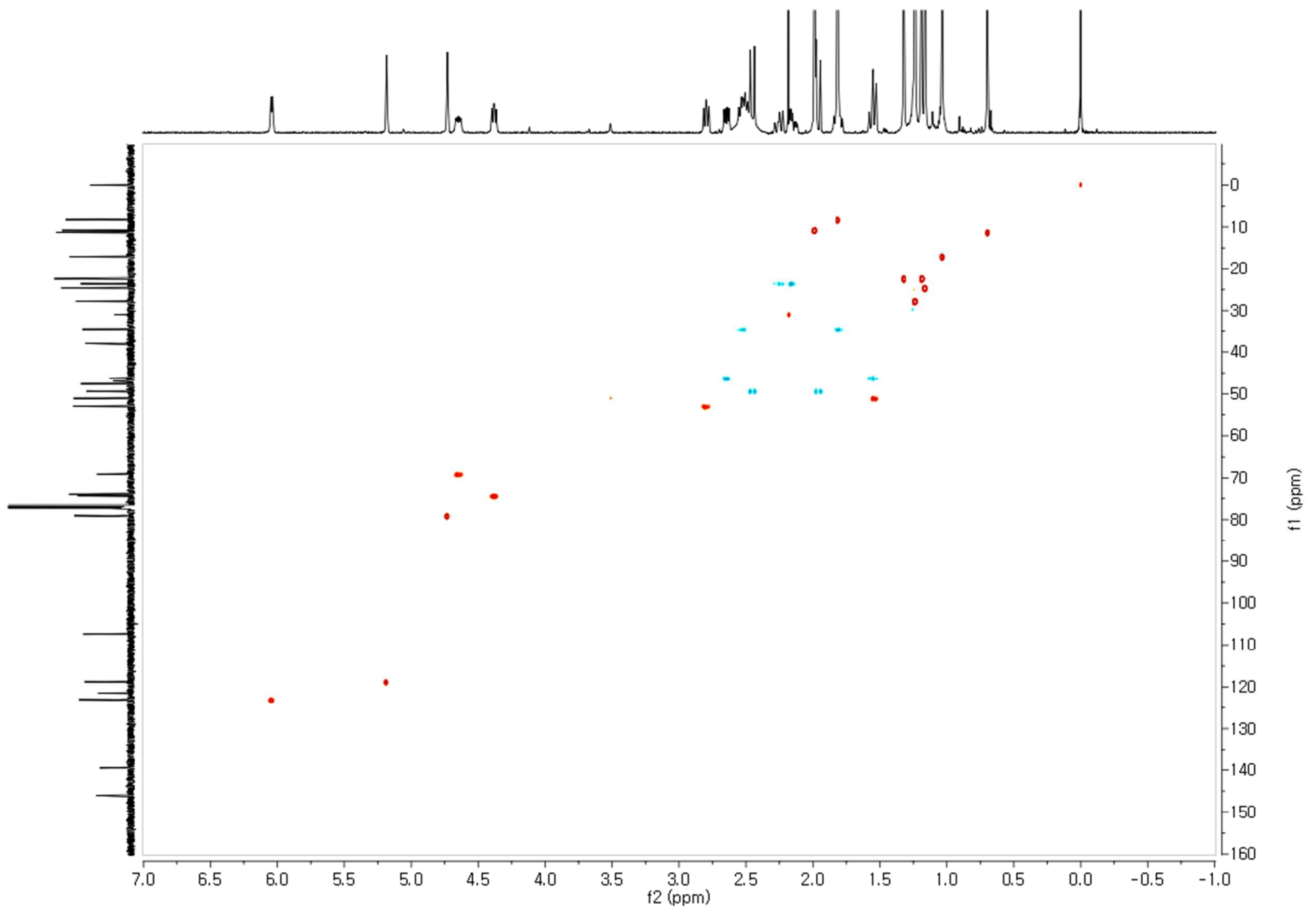


S4. HMBC NMR spectrum $\left(\mathrm{CDCl}_{3}\right)$ antrolactone A (1).

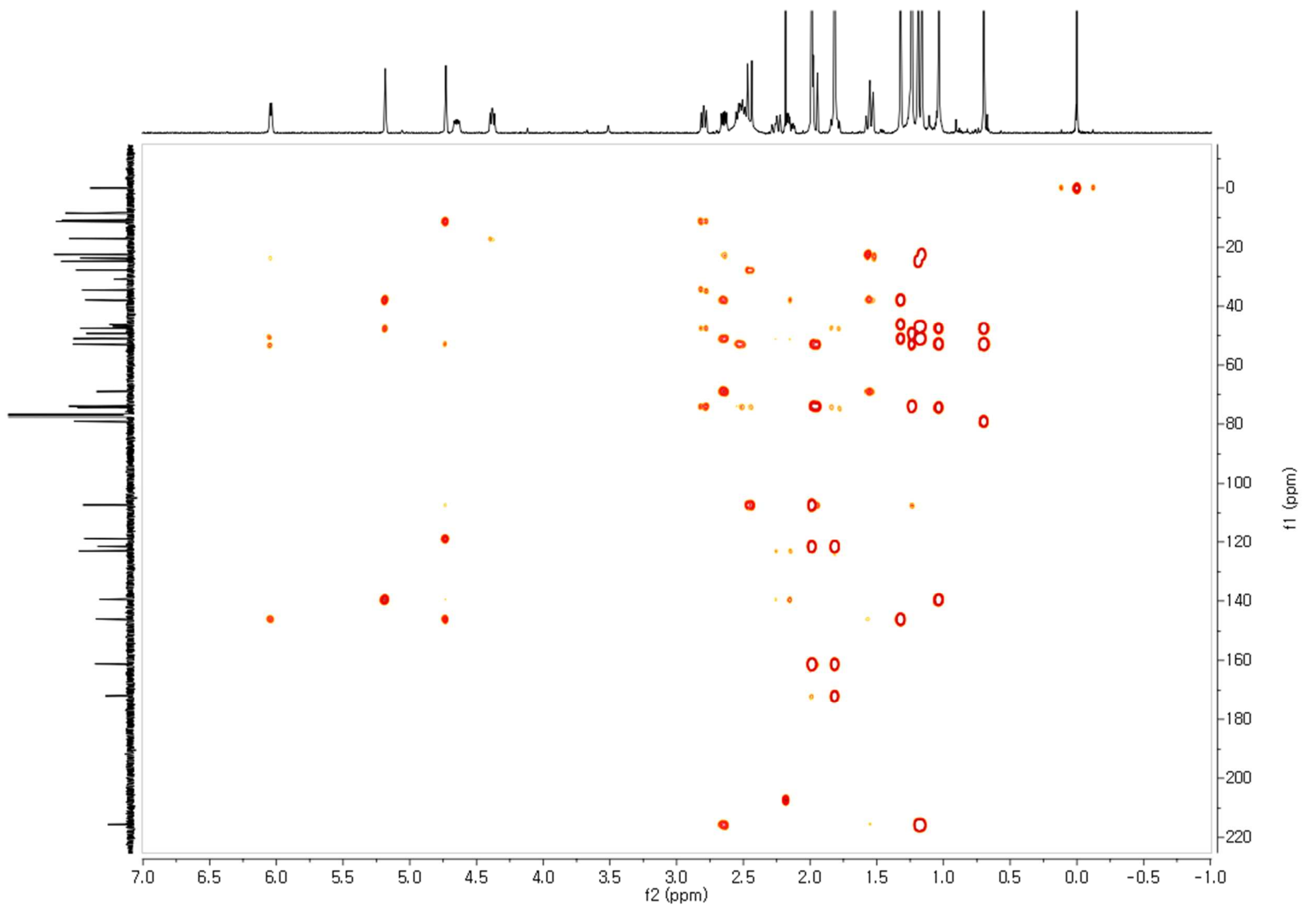


S5. COSY NMR spectrum $\left(\mathrm{CDCl}_{3}\right)$ antrolactone $\mathrm{A}(\mathbf{1})$.

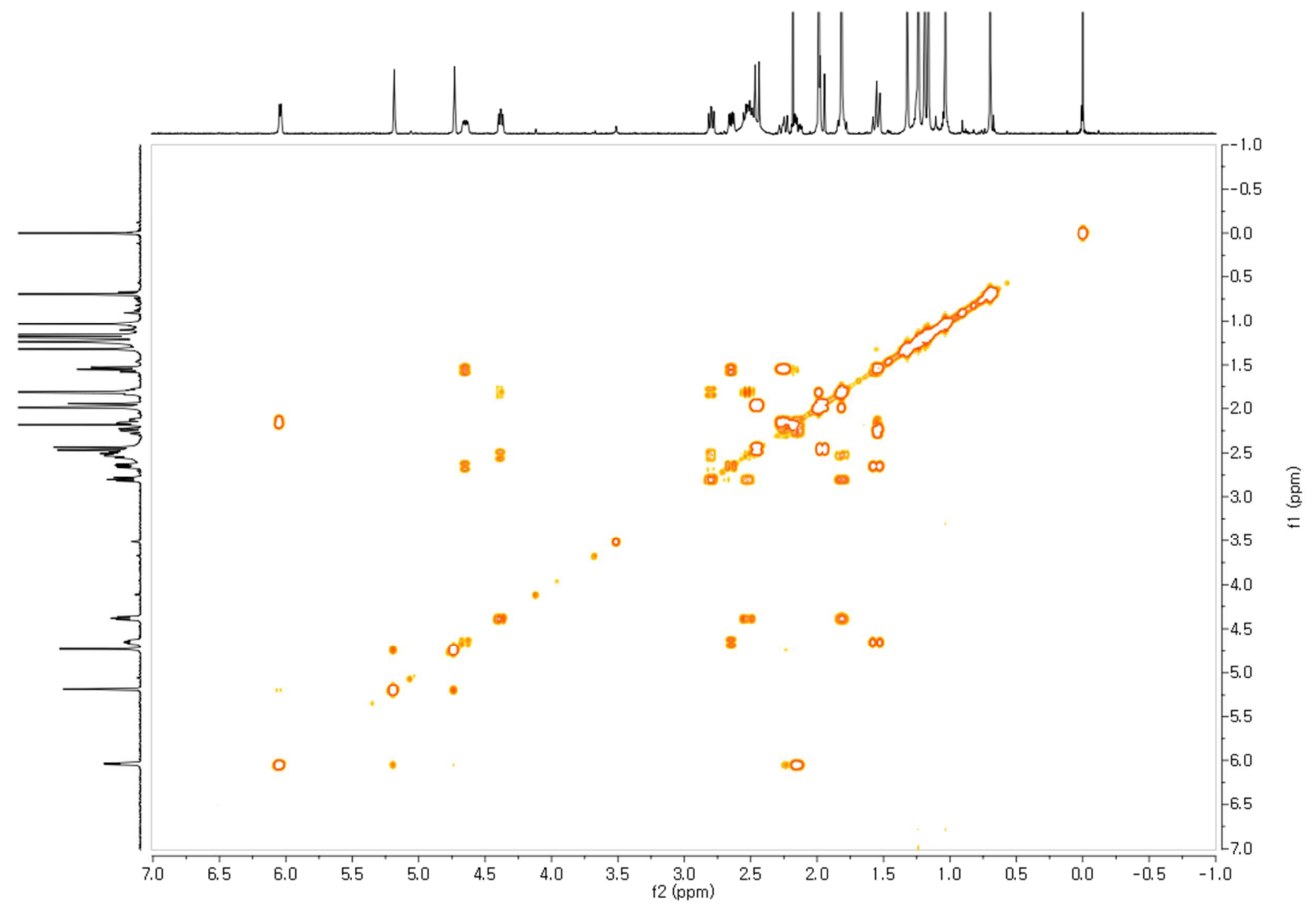


S6. NOESY NMR spectrum $\left(\mathrm{CDCl}_{3}\right)$ of antrolactone A (1).

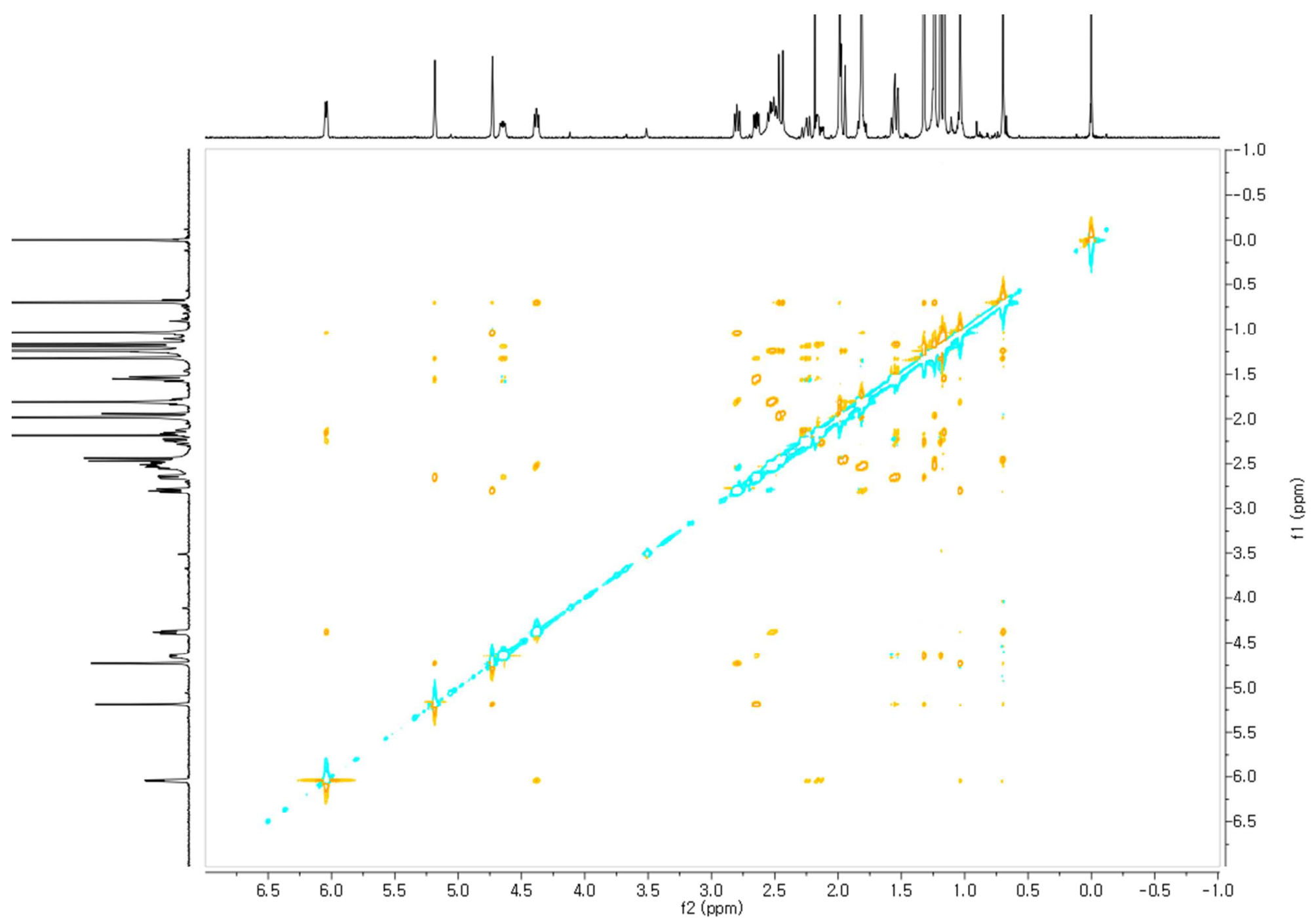


S7. HRESIMS spectrum of antrolactone A (1).

Tolerance $=5.0$ PPM / DBE: $\min =-1.5, \max =50.0$

Selected filters: None

Monoisotopic Mass, Even Electron lons

247 formula(e) evaluated with 1 results within limits (up to 50 best isotopic matches for each mass)

\begin{tabular}{l|c|c|c|c|l|l|l|l|l|l|}
\hline Mass & Calc. Mass & MOA & PPM & DBE & Formula & i-FIT & C & H & N & O \\
\hline 571.2906 & 571.2907 & -0.1 & -0.2 & 11.5 & C32 H43 09 & 5546965.5 & 32 & 43 & & 9
\end{tabular}

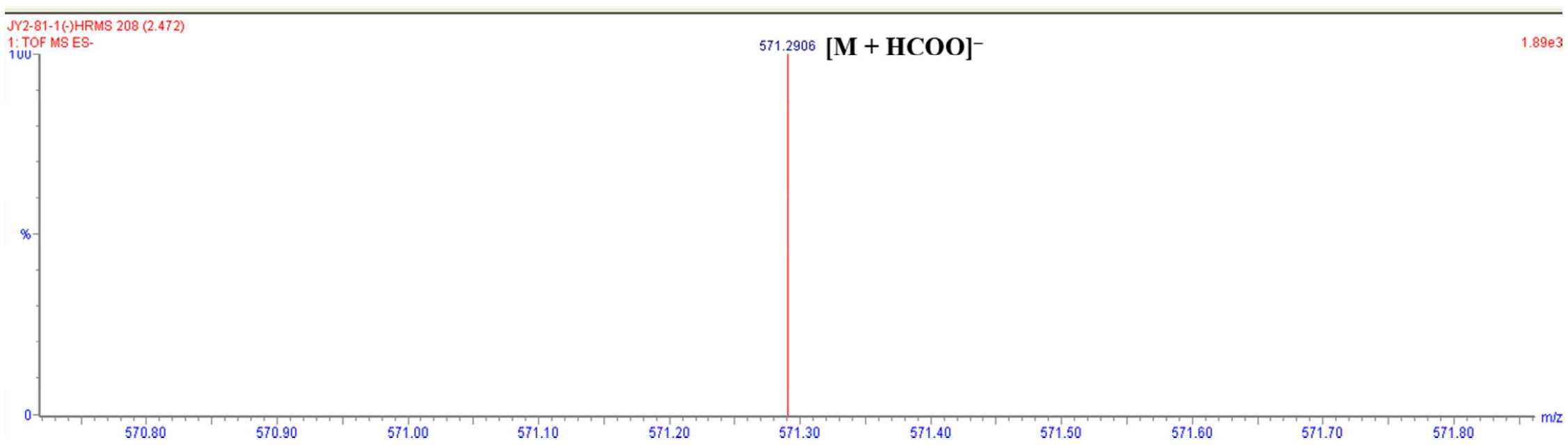


S8. ${ }^{1} \mathrm{H}$ NMR spectrum (500 MHz, $\mathrm{CDCl}_{3}$ ) of antrolactone B (2).

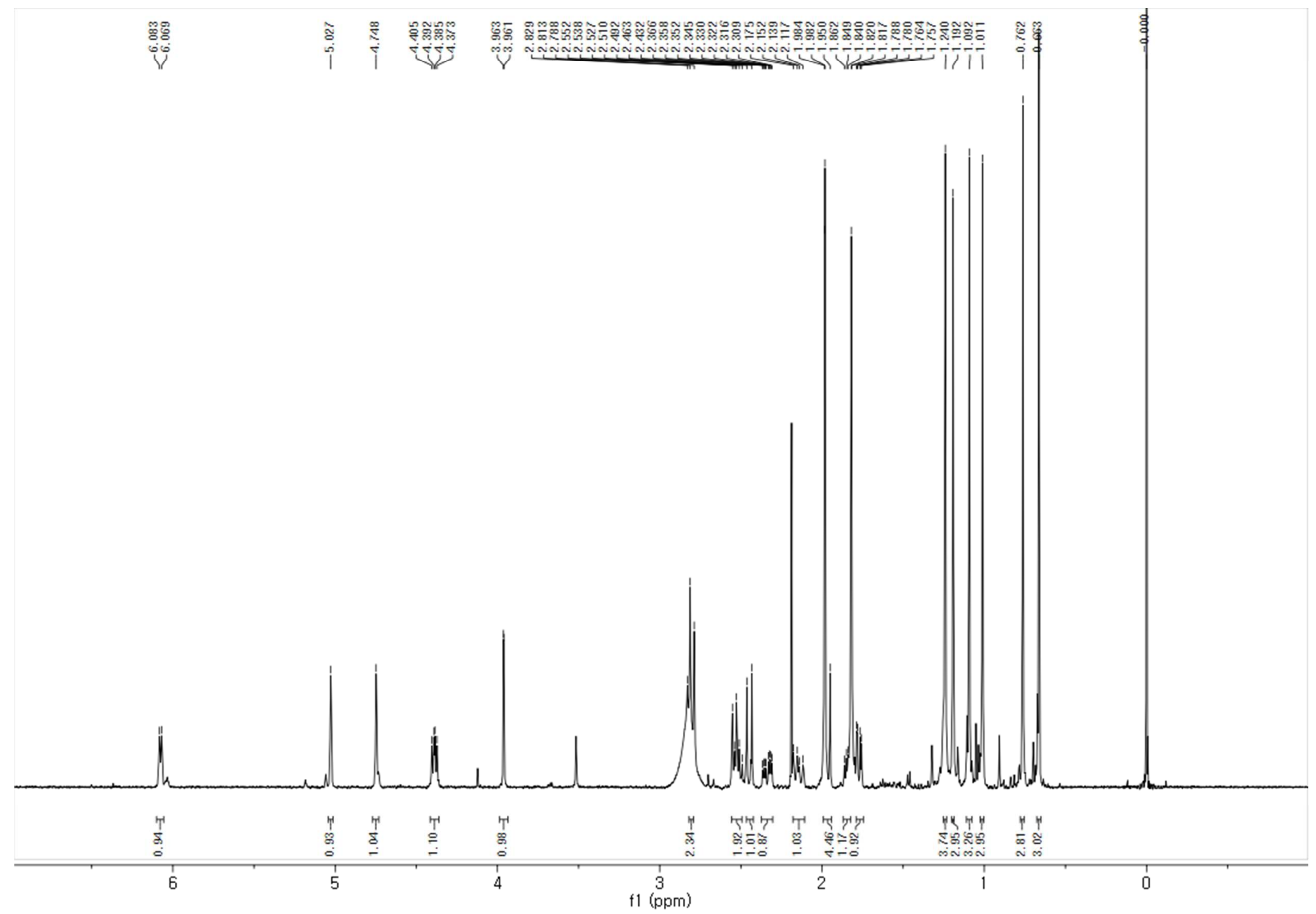


S9. ${ }^{13} \mathrm{C}$ NMR spectrum (125 MHz, $\mathrm{CDCl}_{3}$ ) of antrolactone B (2).

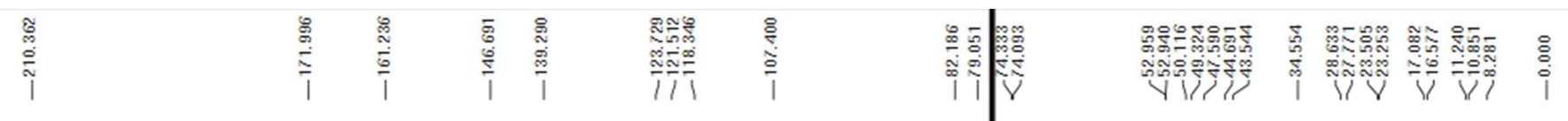

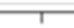

200

180

160

140

120

$1(\mathrm{ppm})$

80

60

40

20

0 
S10. HSQC NMR spectrum $\left(\mathrm{CDCl}_{3}\right)$ of antrolactone B (2).

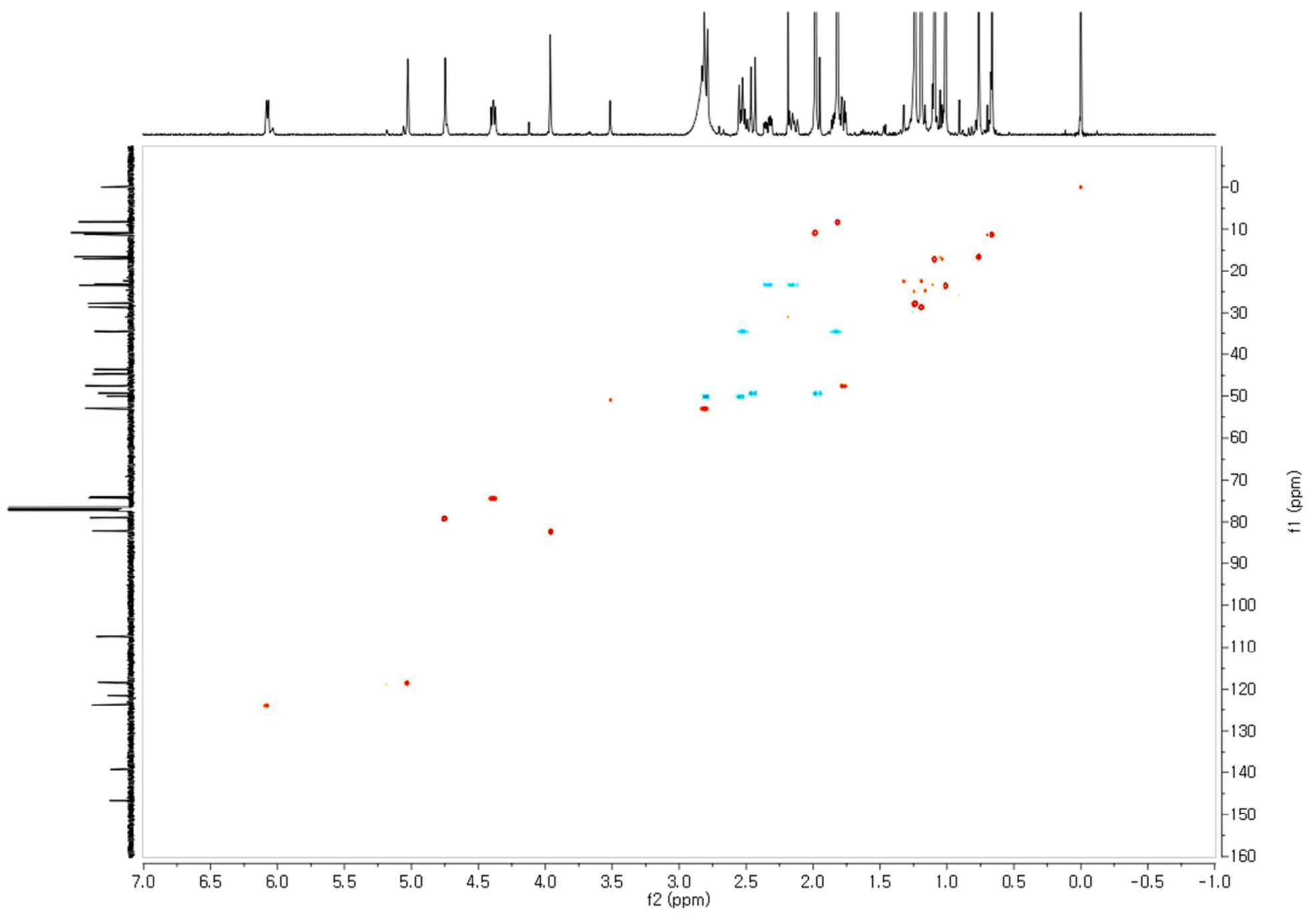


S11. HMBC NMR spectrum $\left(\mathrm{CDCl}_{3}\right)$ of antrolactone B (2).

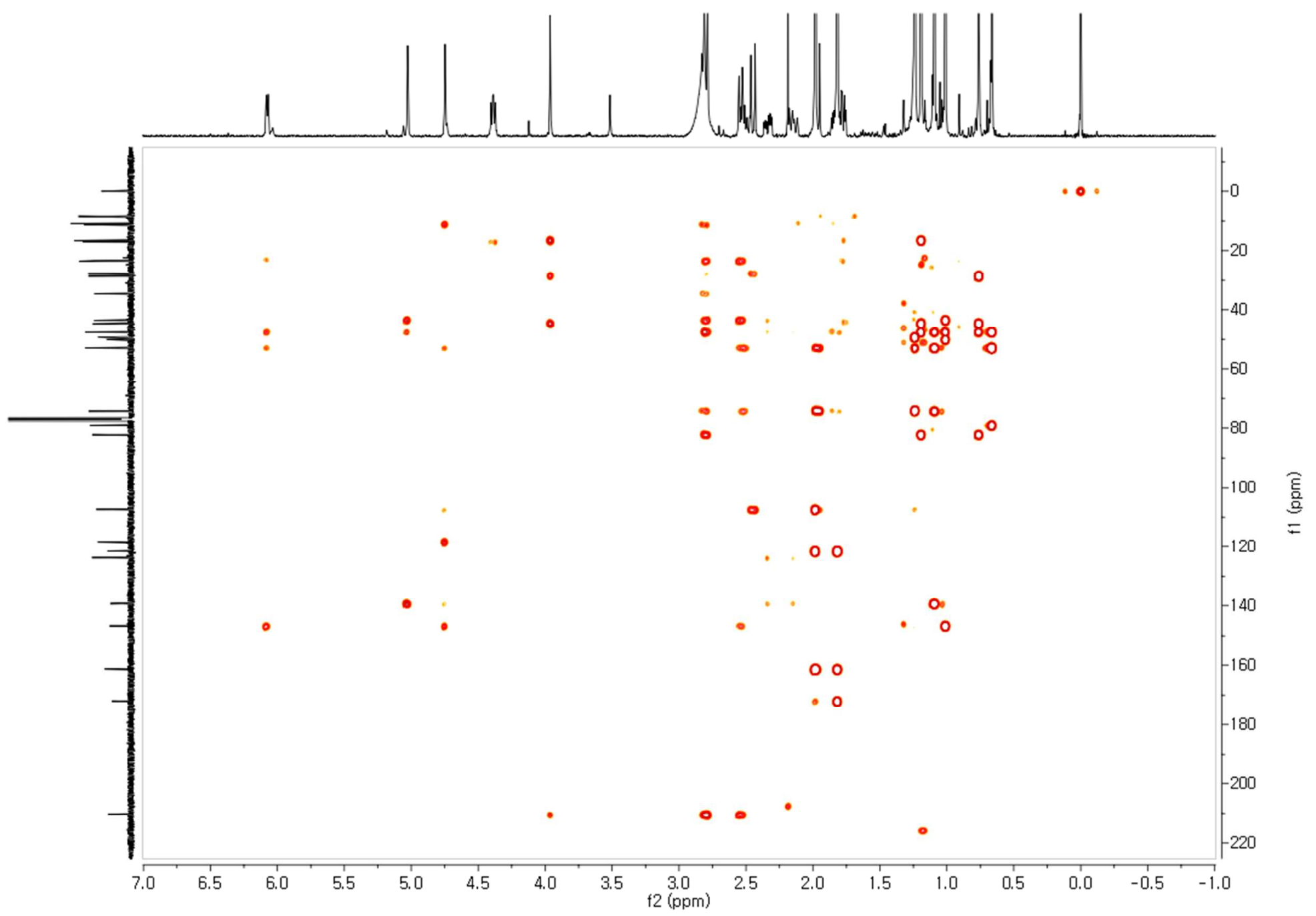


S12. COSY NMR spectrum $\left(\mathrm{CDCl}_{3}\right)$ of antrolactone B (2).

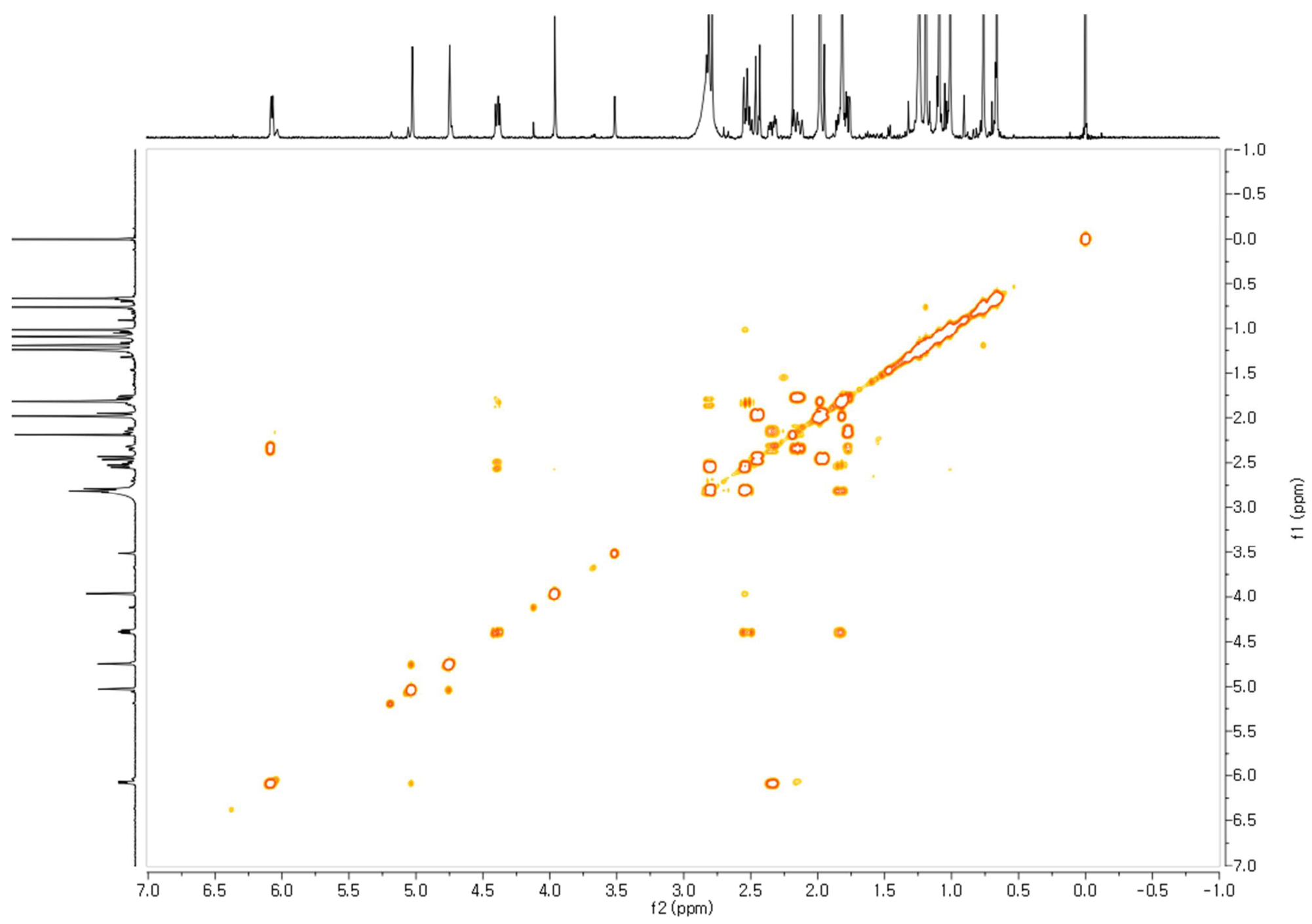


S13. NOESY NMR spectrum $\left(\mathrm{CDCl}_{3}\right)$ of antrolactone B (2).

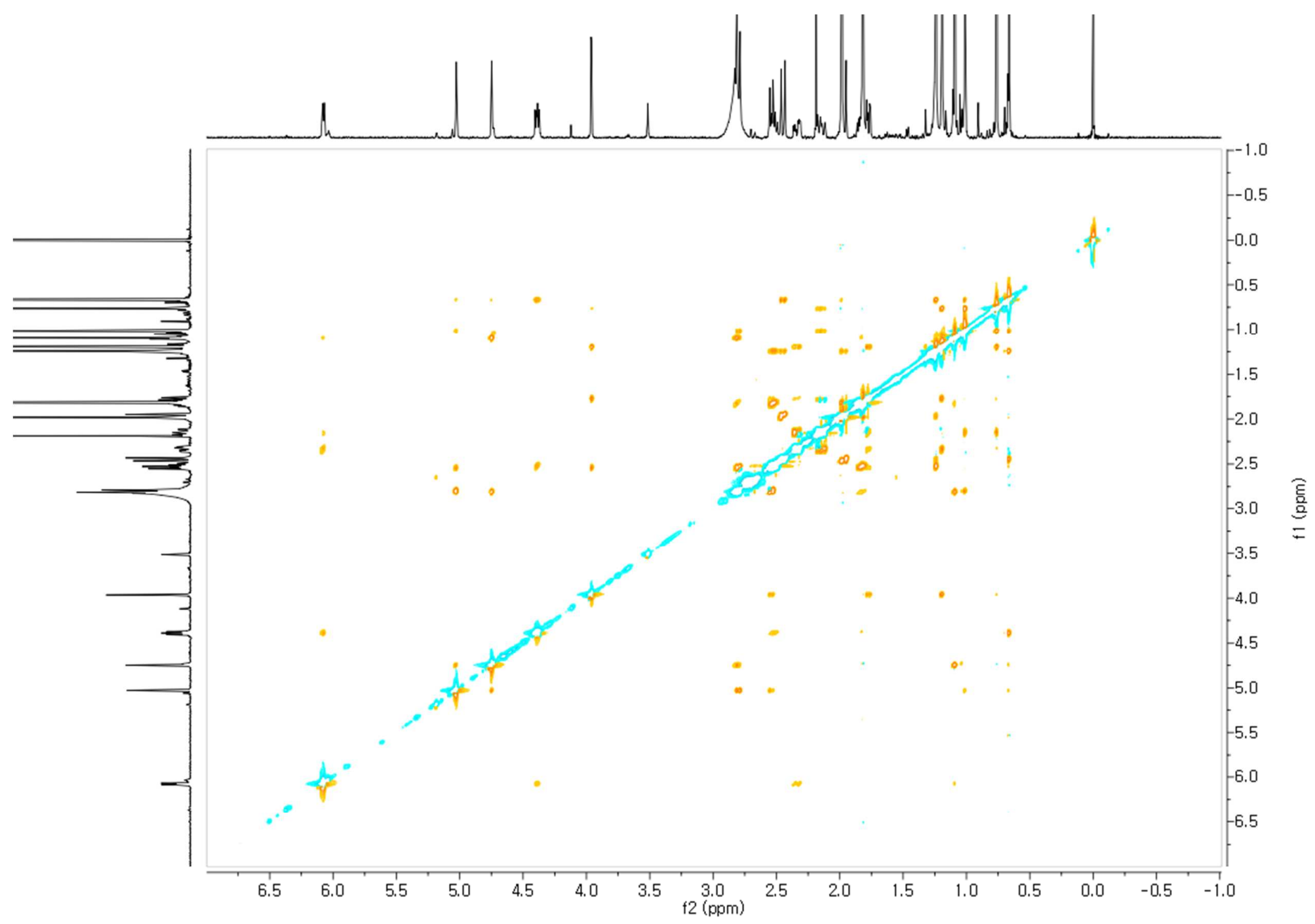


S14. HRESIMS spectrum of antrolactone B (2).

Tolerance $=5.0$ PPM / DBE: $\min =-1.5, \max =50.0$

Selected filters: None

Monoisotopic Mass, Even Electron lons

247 formula[e] evaluated with 1 results within limits (up to 50 best isotopic matches for each mass)

\begin{tabular}{|l|c|c|c|c|l|l|c|c|c|c|}
\hline Mass & Calc. Mass & mDa & PPM & DBE & Formula & i-FIT & C & H & N & O \\
\hline 571.2913 & 571.2907 & 0.6 & 1.1 & 11.5 & C 32 H43 09 & 5547583.5 & 32 & 43 & 9
\end{tabular}

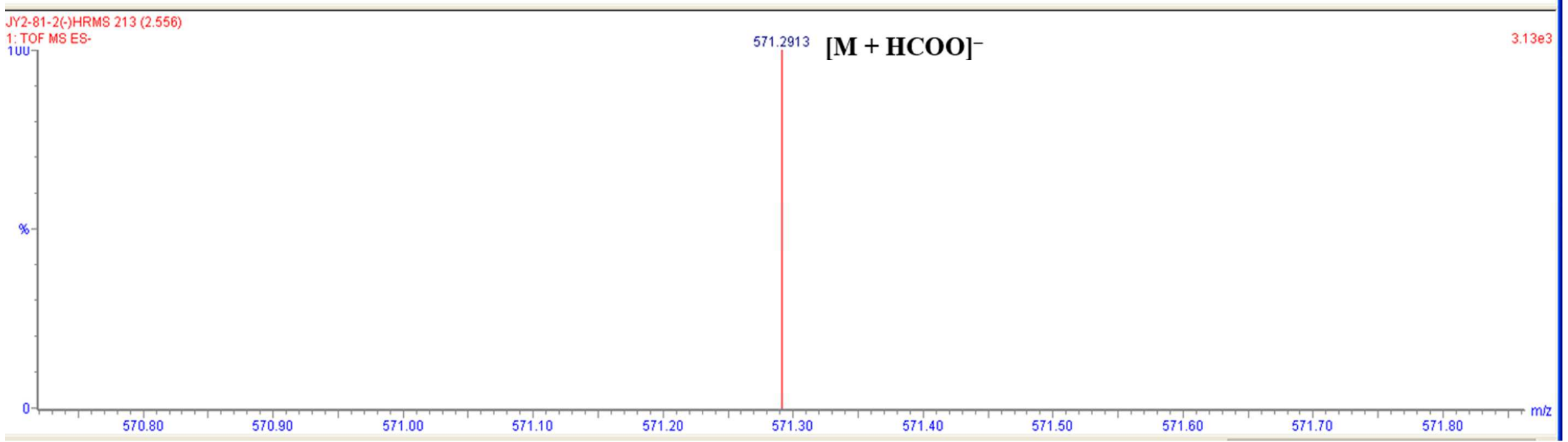


S15. ${ }^{1} \mathrm{H}$ NMR data of compound $\mathbf{3}$.

Polyporenic acid $C(3):{ }^{1} \mathrm{H}$ NMR (pyridine- $\left.d_{5}, 500 \mathrm{MHz}\right) \delta_{\mathrm{H}} 5.60(1 \mathrm{H}, \mathrm{d}, J=6.5 \mathrm{~Hz}, \mathrm{H}-7)$, $5.37(1 \mathrm{H}, \mathrm{d}, J=6.0 \mathrm{~Hz}, \mathrm{H}-11), 5.00(1 \mathrm{H}, \mathrm{s}, \mathrm{H}-31 \mathrm{a}), 4.86(1 \mathrm{H}, \mathrm{s}, \mathrm{H}-31 \mathrm{~b}), 4.55(1 \mathrm{H}, \mathrm{t}, J=6.0$ Hz, H-16), 2.95 (1H, m, H-20), 2.89 (1H, m, H-17), 1.64 (1H, m, H-5), 1.48 (3H, s, H-30), 1.15 (6H, s, H-19, H-29), 1.08 (3H, s, H-18), 1.07 (3H, s, H-28), 1.01 (3H, d, J=6.5 Hz, H27), $1.00(3 \mathrm{H}, \mathrm{d}, J=6.5 \mathrm{~Hz}, \mathrm{H}-26)$ 
S16. BMMs were treated with vehicle or indicated concentrations of 1-3 in the presence of RANKL and M-CSF for 4 days. Cell viability was measured by XTT assay.

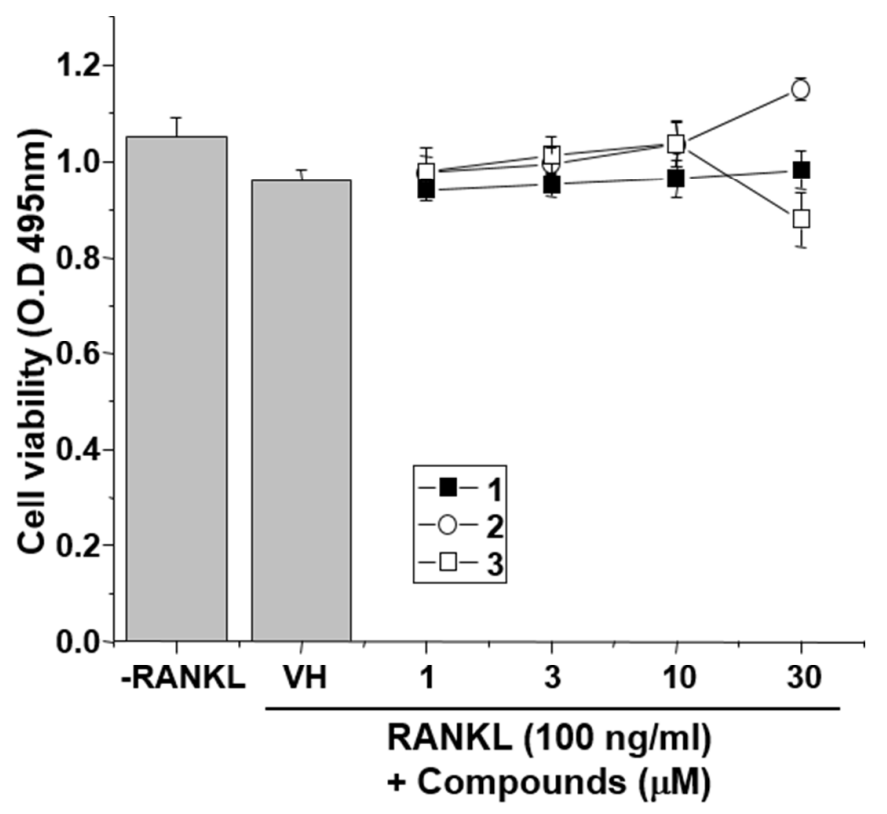

